\title{
Small Modular Reactor Reactivity Disturbance Suppression Method Based on Core Coolant Flow Control
}

Hongyun Xie ( $\sim$ hyxie_cgn@163.com )

China Nuclear Power Engineering Co., Ltd

Qizhi Duan

China Nuclear Power Engineering Co., Ltd

Jialin Ping

China Nuclear Power Engineering Co., Ltd

Chao Lu

China Nuclear Power Engineering Co., Ltd

Liming Zhang

China Nuclear Power Engineering Co., Ltd

Shuqiang Li

China Nuclear Power Engineering Co., Ltd

\section{Research Article}

Keywords: Small modular reactor, Reactivity disturbances suppression, Reactor power disturbance, Doppler effect.

Posted Date: July 16th, 2021

DOl: https://doi.org/10.21203/rs.3.rs-719201/v1

License: (9) This work is licensed under a Creative Commons Attribution 4.0 International License. Read Full License 


\title{
Small modular reactor reactivity disturbance suppression method based on core coolant flow control
}

\author{
Hongyun Xie*, Qizhi Duan, Jialin Ping, Chao Lu, Liming Zhang, Shuqiang Li \\ State Key Laboratory of Nuclear Power Safety Monitoring Technology and Equipment, China Nuclear Power Engineering Co., Ltd., Shenzhen 518172, \\ China \\ Corresponding author: Hongyun Xie (e-mail: hyxie_cgn@163.com).
}

This work was supported in part by the Guangdong Basic and Applied Basic Research Foundation (project No.2019B1515120060), and in part by the Open Funds of State Key Laboratory of Nuclear Power Safety Monitoring Technology and Equipment.

\begin{abstract}
Nuclear reactors may suffer from various disturbances during operation. These disturbances can cause core power deviates from the set parameters, and affect the power level of reactors. Due to the limited internal space of the reactor, the number of control rods is small. It is difficult to set up control rod groups dedicated to reactive compensation for modular reactor of medium or small size. Therefore, it is necessary to study a set of reactivity compensation measures that do not rely on control rods according to the actual needs of modular reactors to compensate for the power deviation caused by reactivity disturbances. A disturbance suppression method based on coolant flow control is proposed in the study. This method takes advantage of the Doppler effect of the coolant temperature, and changes the coolant flow rate to affect its temperature when reactive disturbances occur, thereby compensating for fluctuations of reactivity. Numerical experiments show that this method can effectively suppress the power deviation caused by reactive disturbances, and has engineering application value.
\end{abstract}

INDEX TERMS Small modular reactor; Reactivity disturbances suppression; Reactor power disturbance; Doppler effect.

\section{INTRODUCTION}

Small modular nuclear reactors have been developed widely during recent years. For ensuring safe and effective operation, a desirable core power control system is necessary for small modular reactors $[1,2]$. The core power control system should have two critical functions: core power level control (figure 1), and reactivity disturbances suppression [3].

For the core power level control, there are some advanced methods are developed gradually. Including Model Reference adaptive control [4-5], model predictive control [6-8], fuzzy logical control [9], linear quadratic gaussian with loop transfer recovery [10-11], sliding mode control [12-14], and fractional order control $[15,16]$. Some of them have gradually become mature methods for power level control. Besides on the adjustment of power level, nuclear reactors may suffer from various reactivity disturbances during operation. These disturbances can cause core power deviates from the set parameters, and affect the power level of reactors. However, for modular reactors, the internal space is limited, so that there may be not enough control rods for reactivity disturbances suppression in modular reactors. Therefore, it is necessary to study a set of reactivity compensation measures that do not rely on control rods according to the actual needs of modular reactors to compensate for the power deviation caused by reactivity disturbances.

For the purpose of reactivity disturbances suppression in modular nuclear reactors, a reactivity disturbances suppression method without control rods movement or boron concentration adjustment was developed in this work. Considering the Doppler effect of coolant and fuel temperature, a disturbance suppression method based on coolant flow control is proposed. This method changes the coolant flow rate to affect its temperature when reactive disturbances occur, thereby compensating for fluctuations of reactivity.

The rest of this paper is organized as follows: Firstly, a common NPP dynamic model containing neutron kinetic and 
thermal-hydraulic was built; and then the formulation of control problem about uncertain reactivity disturbances was presented; after that, based on the analysis of zero-poles, the disturbance suppression scheme based on flow control was designed. at last, the effectiveness of the reactivity disturbances suppression method is verified.

\section{Reference Design}

In order to establish a simulation model and verify the effectiveness of the method in this study, the SNCLFR-100 is regarded as the reference design.

SNCLFR-100 is a typical modular reactor with only a small number of control rod groups for power level adjustment, and no boron adjustment equipment or control rods for reactivity compensation. Although the primary circuit coolant of the SNCLFR-100 is lead, the method proposed in this article is also applicable to coolants such as water and carbon dioxide.

SNCLFR-100 is a $100 \mathrm{MW}_{\text {th }}$ lead-cooled small modular reactor with a passive cooling feature to both normal and abnormal operations, was proposed by University of Science and Technology of China (USTC). The reactor is well suited as a remote power source because of its compact size, as well as because it has a refueling interval of 10 years without assembly reconfiguration. The major parameters of SNCLFR-100 are listed in Table 1 [17].

Table 1. Major parameters of SNCLFR-100

\begin{tabular}{c|c}
\hline Parameters & Value \\
\hline Reactor type & SMR \\
\hline Fuel type & MOX \\
Core power & $100 \mathrm{MW}$ (thermal) \\
Steam generator & Straight shell-tube type \\
Primary cooling model & Fully natural circulation \\
Core inlet temperature & $400^{\circ} \mathrm{C}$ \\
Core outlet temperature & $480^{\circ} \mathrm{C}$ \\
Design volume flow flux & $8528 \mathrm{~kg} / \mathrm{s}$ \\
\hline
\end{tabular}

The primary cooling model of SNCLFR-100 is fully natural circulation, which is not conducive to core coolant flow control. Therefore, this article improves the design of the SNCLFR-100, which is named SNCPWR-100, four non-seal centrifugal pumps are used to regulated the flow, and the type of coolant changed from lead to pressurized water with $15.5 \mathrm{MPa}$. The major parameters of SNCPWR-100 are listed in Table 2.
Table 2. Major parameters of improved SNCPWR-100

\begin{tabular}{c|c}
\hline Parameters & Value \\
\hline Reactor type & SMR \\
\hline Fuel type & MOX \\
Core power & $100 \mathrm{MW}$ (thermal) \\
Steam generator & Straight shell-tube type \\
Primary cooling model & non-seal centrifugal pump \\
Operating pressure & $15.5 \mathrm{MPa}$ \\
Core inlet temperature & $292.4^{\circ} \mathrm{C}$ \\
Core outlet temperature & $327.6^{\circ} \mathrm{C}$ \\
Design volume flow flux & $1200 \mathrm{~m}^{3} / \mathrm{h}$ \\
\hline
\end{tabular}

The structure of SNCPWR-100 simulation model is shown schematically in figure 1 . For the verification, three components of the model structure are considered: reactor core, the primary loop and one-through steam generators (OTSGs). The second loop and other auxiliary components are neglected. In the simplified model, two couple of temperature and pressure boundaries are presented for structure integrity.

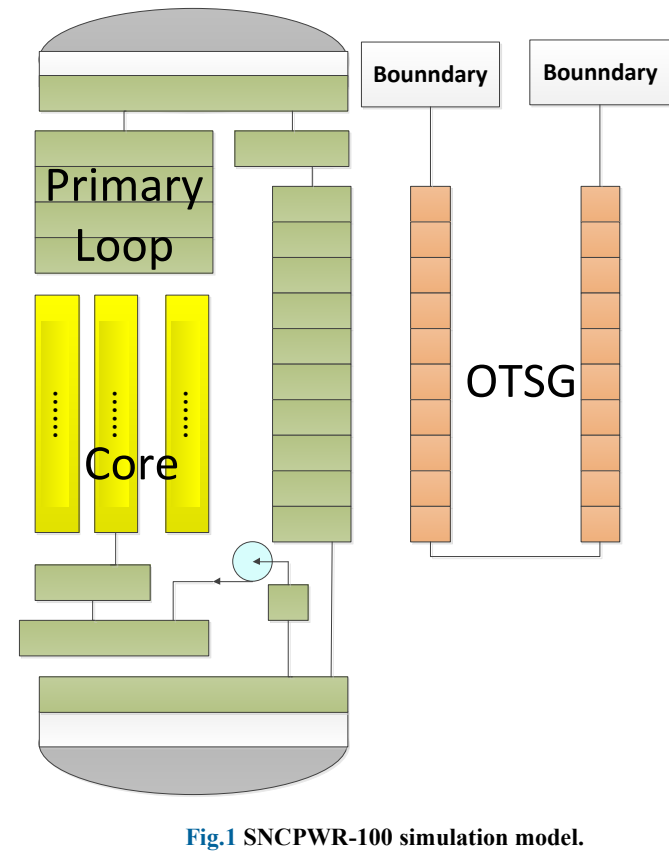

\section{Modeling}

The following lists the calculation equations of neutron kinetic and Thermal-hydraulic models, and the detailed deduction of these equations was presented in the paper of Liming Zhang [3].

\section{A. Neutron Kinetic}

The linearized neutron kinetic model as follows: 
$\frac{d \Delta n(t)}{d t}=\frac{\delta_{0}-\beta}{\Lambda} \Delta n(t)+\frac{n_{0}}{\Lambda} \Delta \delta(t)+\sum_{i=1}^{6} \lambda_{i} \Delta c_{i}(t)$

$\frac{d \Delta c_{i}(t)}{d t}=\frac{\beta_{i}}{\Lambda} \Delta n(t)-\lambda_{i} \Delta c_{i}(t)$

And the total reactivity is the control rod reactivity combined with the feedbacks described as follows:

$$
\delta(t)=\delta_{\text {rod }}+\alpha_{f}\left(T_{f}-T_{f 0}\right)+\alpha_{\text {coolant }}\left(T_{p}-T_{p 0}\right)
$$

\section{B. Thermal-hydraulic}

\section{(1) Core}

The increment form of core heat transfer process can be given as:

$$
\rho_{f} V_{f} C_{f} \frac{d \Delta T_{f}}{d t}=\Delta P-U_{t} A_{c}\left(\Delta T_{f}-\Delta T_{c}\right)
$$

$\rho_{c} V_{c} C_{p} \frac{d \Delta T_{c}}{d t}$

$$
=U_{t} A_{c}\left(\Delta T_{f}-\Delta T_{c}\right)-G_{p} C_{p}\left(\Delta T_{\text {cout }}-\Delta T_{\text {cin }}\right)
$$

\section{(2) Steam Generator}

The increment form of steam generators heat transfer process can be given as:

$$
\begin{aligned}
& \rho_{\text {sgp }} V_{\text {sgp }} C_{p} \frac{d \Delta T_{\text {sgp }}}{d t} \\
& =G_{p} C_{p}\left(\Delta T_{\text {sgpin }}-\Delta T_{\text {sgpout }}\right)-U_{s g} A_{s g} \Delta T_{s g \Delta} \\
& \rho_{s g s} V_{s g s} C_{s} \frac{d \Delta T_{s g s}}{d t}= \\
& G_{s} C_{s}\left(\Delta T_{f w}-\Delta T_{\text {sgsout }}\right)+U_{s g} A_{s g} \Delta T_{\text {sg }}
\end{aligned}
$$

\section{Coupling Process}

In the SMR model, $(\Delta n, \Delta P),\left(\Delta T_{\text {cout }}, \Delta T_{\text {sgpin }}\right)$, and $\left(\Delta T_{\text {sgpout }}, \Delta T_{\text {cin }}\right)$ are three pairs of couple variables:

$$
\begin{aligned}
& \Delta P=\frac{\Sigma_{f} \Delta n U_{e} \mathrm{~B}}{3.125 \times 10^{10}} \Delta n \\
& \Delta T_{\text {sgpin }}+\tau_{1} \frac{d \Delta T_{\text {sgpin }}}{d t}=\Delta T_{\text {cout }} \\
& \Delta T_{\text {cin }}+\tau_{2} \frac{d \Delta T_{\text {cin }}}{d t}=\Delta T_{\text {sgpout }}
\end{aligned}
$$

MATLB/Simulink software is used to simulate the above model, and the figure 2 is the simulation flow chart of the model.

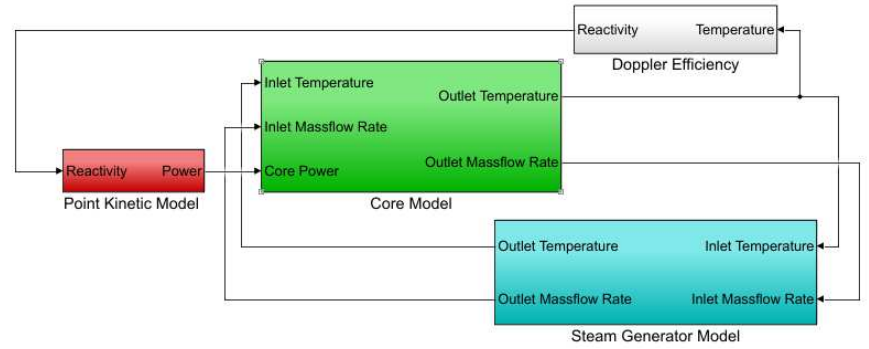

Fig.2 Open-loop model of reactor neutron dynamics coupled with thermal hydraulics

The simulation results are shown in figure 3 to figure 5 . In the case of power perturbation, the steady-state power of the reactor changes, although the reactor has good sub-stability (lost $5 \%$ of our power). If reactivity compensation is not carried out, the output power will be affected.

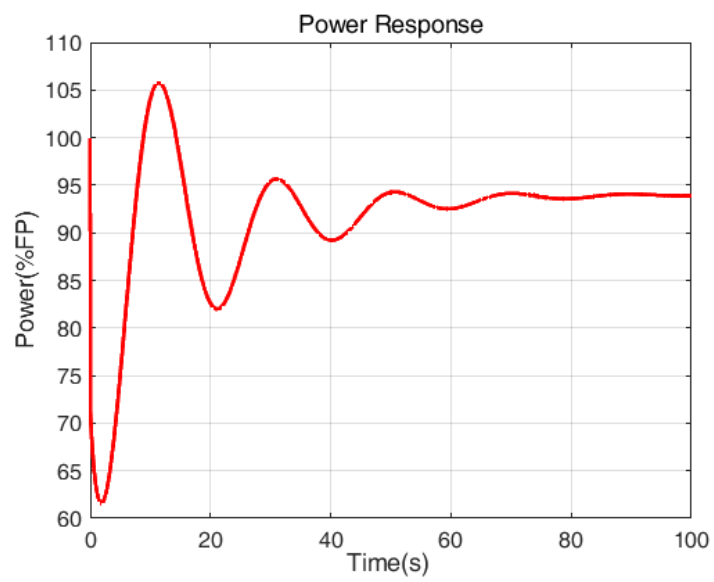

Fig.3 Power open-loop response under reactivity perturbation

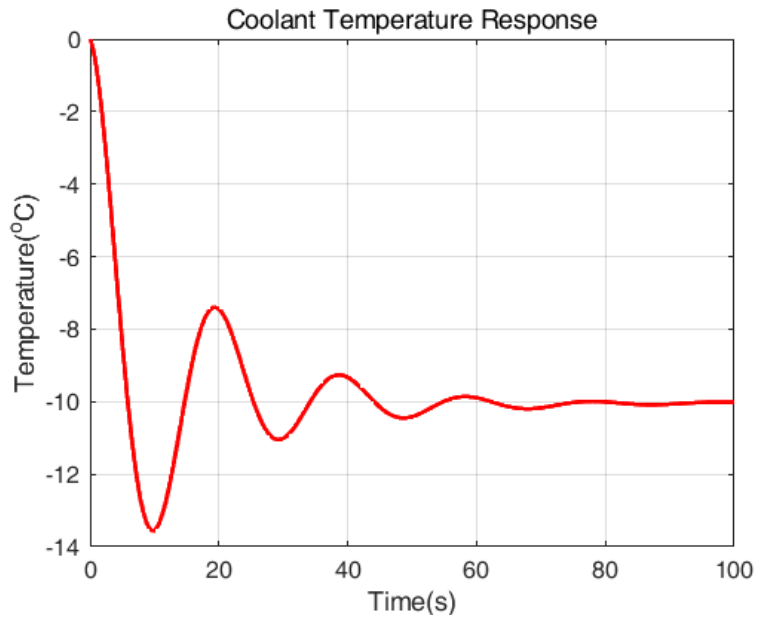

Fig.4 Core coolant temperature open-loop response under reactive perturbation 


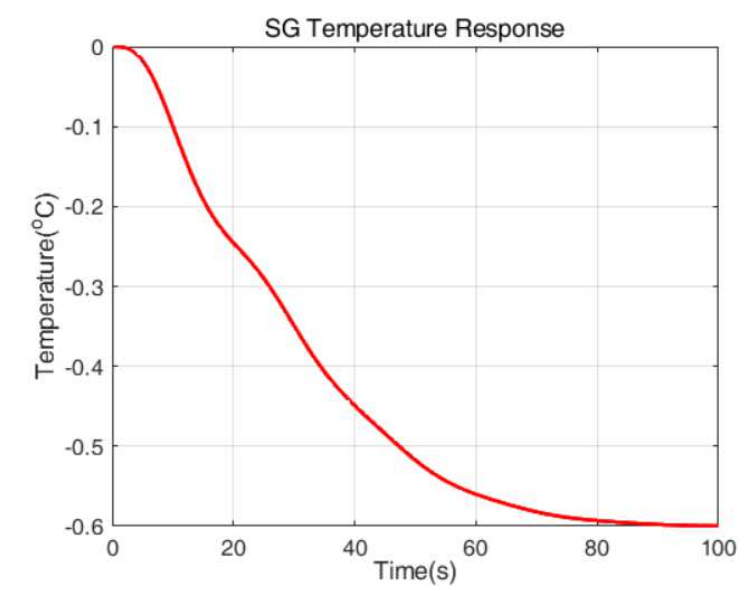

Fig.5 Open loop response of steam generator outlet temperature under reactive

perturbation

IV. Design of Disturbance Suppression Scheme Based on Flow Control

Due to the small number of control rod groups in modular reactors, additional reactivity compensation schemes need to be developed to suppress the effects of reactivity disturbances. Considering the significant negative temperature Doppler feedback effect in the core coolant, the reactivity can be compensated from the temperature by changing the core coolant temperature in reverse to maintain the constant power level.

The overall disturbance suppression strategy designed is shown in figure 6.

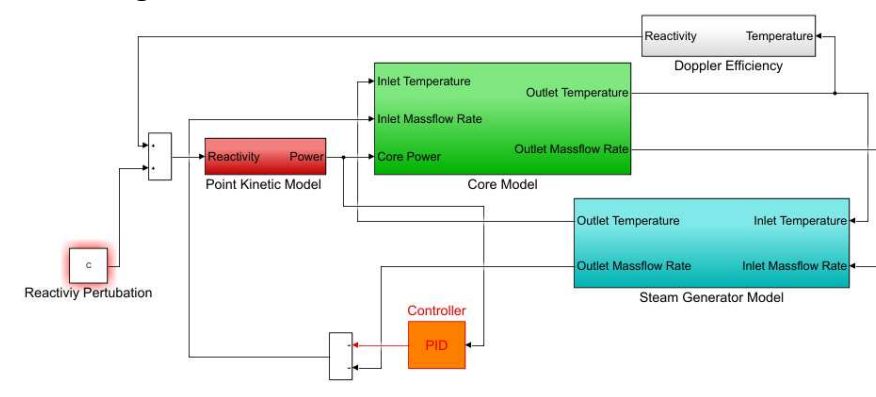

Fig.6 The overall strategy model of disturbance suppression

In this strategy, the reactor core power is used as feedback, and the core flow rate is changed through the regulation of the controller, so as to affect the coolant temperature in the core, and then the introduction of reactivity is changed through the Doppler effect. And the controller adopts common PID controller as follow:

$\Delta \mathrm{G}=K_{p} \Delta \mathrm{P}+\mathrm{K}_{i} \int_{0}^{t} \Delta \mathrm{Pdt}$

In order to ensure that the reactor power can completely converge to the initial state, the integral coefficient $\mathrm{K}_{i}$ is introduced. In order to speed up the adjustment process, differential terms are not used.

The overall stability of the reactor system can be further ensured by the zero-pole distribution under the action of the above mentioned PID controller, that is, after the addition of the above mentioned PID controller, as is shown in figure 7, all the poles of the system are in the left half-open plane of the complex plane (L.H.C.P) [18].

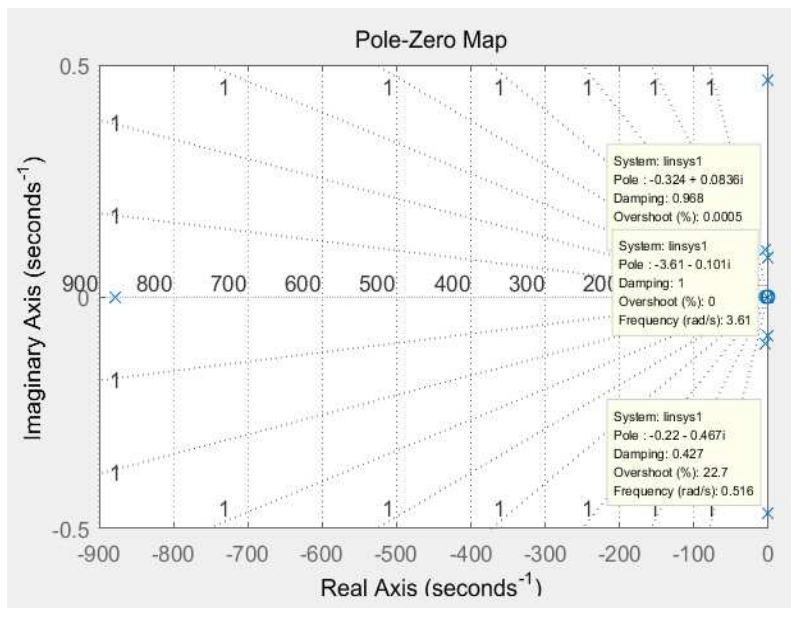

Fig.7 Closed loop pole distribution with the controller

\section{Results and Discussion}

For verifying the effectiveness clearly, two dynamic responses are calculated: one is the response of the model with controller, and the other is the response of the model without controller.

The inhibition effect of the controller designed in this paper on the reactivity disturbance is shown in figure 8 (including power, core temperature and steam generator temperature).

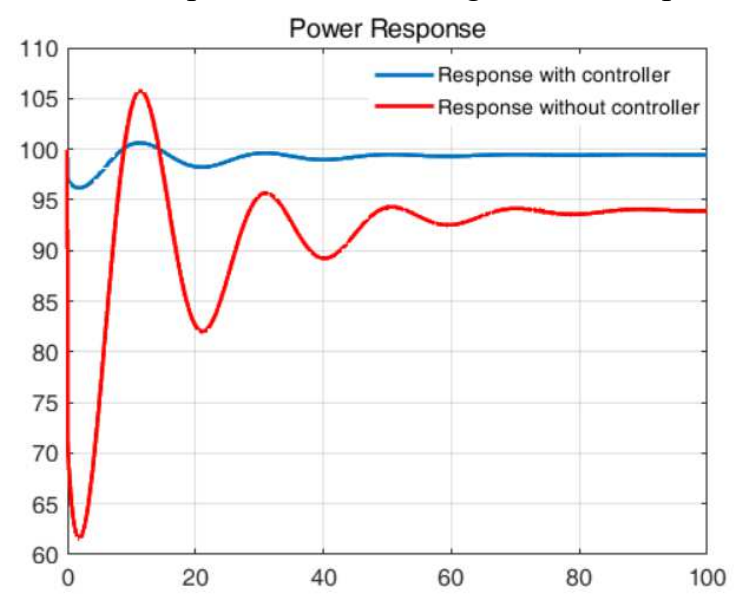

Fig.8 Comparison of power responses with and without controller

Under the perturbation of reactivity, compared with the power response without flow controller, the power response with flow controller has smaller fluctuation range and faster convergence rate, and finally converges to the set value. 
Figure 9 shows the comparison of core coolant temperature response under the condition of reactive disturbance based on flow regulation and under the condition of no regulation. The temperature response amplitude of the core without the controller is large and soon deviates from the set value. The core temperature response with the controller is stable near the set value.

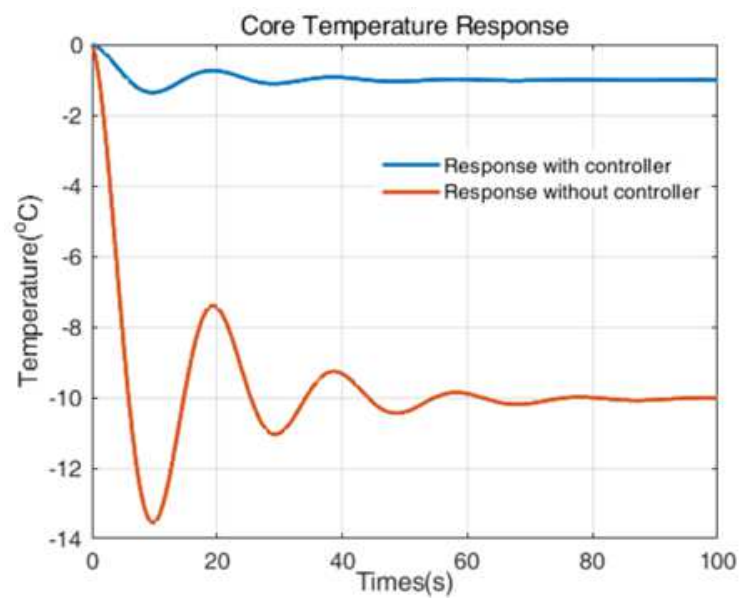

Fig.9 Comparison of core temperature responses with and without controller

As shown in figure 10, without a controller to regulate the flow rate, the temperature response of the steam generator cannot be stabilized at the set value.

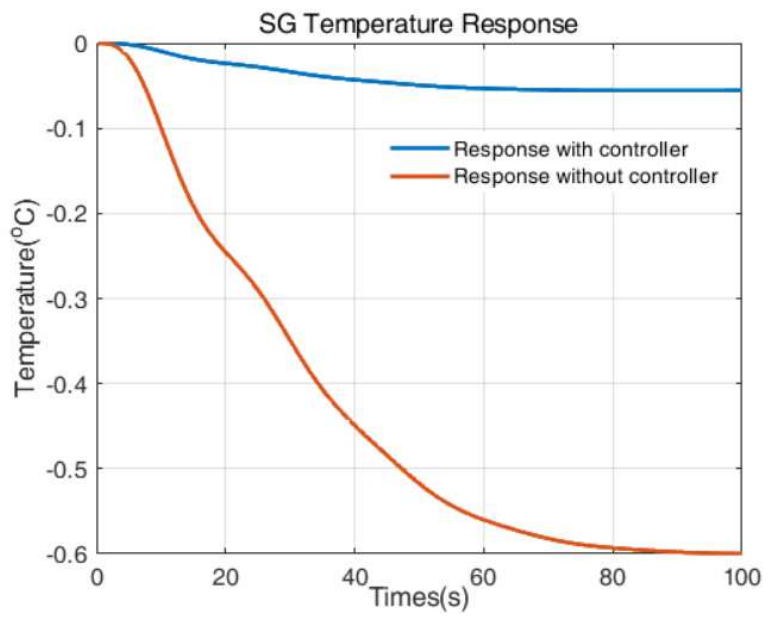

Fig.10 Comparison of SG temperature responses with and without controller

In conclusion, the disturbance suppression scheme based on flow control can effectively regulate the stability of reactor power, core temperature and evaporator temperature.

\section{Conclusion}

When modular nuclear reactors suffer from uncertain reactivity disturbance, core power may deviate from the set parameters, and affect the power level of reactors. Due to the limited internal space of modular nuclear reactors, the number of control rods is small. It is difficult to set up control rod groups dedicated to reactive compensation for modular reactor of medium or small size.

For this purpose, a reactivity disturbance suppression method is developed. This method does not rely on control rods according to the actual needs of modular nuclear reactors to compensate for the power deviation caused by reactivity disturbances. Considering the Doppler effect of coolant and fuel temperature, a disturbance suppression method based on coolant flow control is proposed in the work. This method changes the coolant flow rate to affect its temperature when reactive disturbances occur, thereby compensating for fluctuations of reactivity. Numerical experiments show that this method can effectively suppress the power deviation caused by reactive disturbances.

In the future, we will continue to verify the disturbance suppression method based on full scope simulator of NPPs. Moreover, the couple mode between the disturbance suppression method and power level control method also needs to be researched.

\section{ACKNOWLEDGMENT}

We thank the great help from Southeast University in this research.

\section{REFERENCES}

[1] Franceschini, F. , Petrovic, B.: 'Advanced operational strategy for the iris reactor: load follow through mechanical shim (mshim). Nuclear Engineering and Design', 2008, 238(12), pp.3240-3252

[2] Ablay, G.: 'A modeling and control approach to advanced nuclear power plants with gas turbines', Energy Conversion and Management, 2013, 76, pp. 899-909.

[3] Zhang, L. , Xie H., Duan Q.: "Power Level Control of Nuclear Power Plant Based on Asymptotical State Observer under Neutron Sensor Fault", Science and Technology of Nuclear Installations 2021.3, pp.1-8.

[4] G. T. Bereznai, "Adaptive Nuclear Reactor Control Based on Optimal Low-Order Linear Models," in IEEE Transactions on Nuclear Science, vol. 20, no. 2, pp. 72-82, April 1973.

[5] Na M G, Dong W J, Sun H S, et al., "Design of a nuclear reactor controller using a model predictive control method," in Ksme International Journal, vol.18, no.12, pp.2080-2094, Dec. 2004.

[6] Man Gyun Na, Dong Won Jung, Sun Ho Shin, Jin Wook Jang, Ki Bog Lee and Yoon Joon Lee, "A model predictive 
controller for load-following operation of PWR reactors," in IEEE Transactions on Nuclear Science, vol. 52, no. 4, pp. 1009-1020, Aug. 2005.

[7] Wang G., Wu J., Zeng B., Xu Z., Wu W., \& Ma X, "Statespace model predictive control method for core power control in pressurized water reactor nuclear power stations," in Nuclear Engineering \& Technology, vol.49, no.3, pp. 134-140, February 2017.

[8] H. Arab-Alibeik and S. Setayeshi, "Improved temperature control of a PWR nuclear reactor using an LQG/LTR based controller," in IEEE Transactions on Nuclear Science, vol. 50, no. 1, pp. 211-218, Feb. 2003.

[9] A. Ben-Abdennour, R. M. Edwards and K. Y. Lee, "LQG/LTR robust control of nuclear reactors with improved temperature performance," in IEEE Transactions on Nuclear Science, vol. 39, no. 6, pp. 2286-2294, Dec. 1992.

[10] Munje, R.K., Patre, B.M., Shimjith, S.R., and Tiwari, A.P.: 'Sliding Mode Control for Spatial Stabilization of Advanced Heavy Water Reactor', Ieee Transactions on Nuclear Science, 2013, 60, (4), pp. 3040-3050.

[11] Ansarifar, G.R., and Akhavan, H.R.J.A.o.N.E.: 'Sliding mode control design for a PWR nuclear reactor using sliding mode observer during load following operation', Ann Nucl Energy, 2015, 75, pp. 611-619.

[12] Etchepareborda, A., and Lolich, J.: 'Research reactor power controller design using an output feedback nonlinear receding horizon control method', Nuclear Engineering and Design, 2007, 237, (3), pp. 268-276.

[13] Eliasi, H., Menhaj, M.B., and Davilu, H.: 'Robust nonlinear model predictive control for nuclear power plants in load following operations with bounded xenon oscillations', Nuclear Engineering and Design, 2011, 241, (2), pp. 533-543. [14] Tai, Y., Hou, S.X., Li, C., and Zhao, F.Y.: 'An improved implicit multiple model predictive control used for movable nuclear power plant', Nuclear Engineering and Design, 2010, 240, (10), pp. 3582-3585.

[15] Ray, S.S., and Patra, A.: 'Numerical simulation for fractional order stationary neutron transport equation using Haar wavelet collocation method', Nuclear Engineering and Design, 2014, 278, pp. 71-85.

[16] Nowak, T.K., Duzinkiewicz, K., and Piotrowski, R.: 'Numerical solution analysis of fractional point kinetics and heat exchange in nuclear reactor', Nuclear Engineering and Design, 2015, 281, pp. 121-130.
[17] Chen, H., Chen, Z., Chen, C., et al.: 'Conceptual design of a small modular natural circulation lead cooled fast reactor snclfr-100', International Journal of Hydrogen Energy, 2016, 41(17), pp.7158-7168.

[18] P. D. Vaswani and D. Chakraborty, "Optimal State Feedback Controller for a Nuclear Reactor," in IEEE Transactions on Nuclear Science, vol. 66, no. 12, pp. 23792387. 